Service social

\title{
Un bilan québécois des quinze premières années du Régime d'assistance publique du Canada (1966-1981) La dimension sociale.
}

\section{Yves Vaillancourt}

Volume 41, numéro 2, 1992

Bilan des réformes

URI : https://id.erudit.org/iderudit/706568ar

DOI : https://doi.org/10.7202/706568ar

Aller au sommaire du numéro

Éditeur(s)

École de service social de l'Université Laval

ISSN

1708-1734 (numérique)

Découvrir la revue

Citer cet article

Vaillancourt, Y. (1992). Un bilan québécois des quinze premières années du Régime d'assistance publique du Canada (1966-1981) La dimension sociale. Service social, 41(2), 19-47. https://doi.org/10.7202/706568ar
Résumé de l'article

L'auteur présente un bilan du Régime d'assistance publique du Canada (RAPC) au cours des années 1966 à 1981, en mettant l'accent sur l'impact de ce programme fédéral à frais partagés sur le développement des programmes québécois de sécurité du revenu et de services sociaux. Après avoir dégagé les principales caractéristiques du RAPC et mis en relief la philosophie sélective inhérente à ce programme, l'auteur examine les interfaces entre le RAPC et le développement des politiques sociales québécoises en distinguant deux sous-périodes. Au cours de la première sous-période, située en amont de la réforme Castonguay-Nepveu et du " chapitre 48 " (1966 à 1971), il ressort que l'harmonisation entre le RAPC et les programmes québécois concernés se réalise sans trop de heurts. Au cours de la deuxième sous-période, située en aval de la réforme Castonguay-Nepveu et du " chapitre 48 » (1971-1981), le RAPC, en raison de sa philosophie sélective, entre en contradiction avec la philosophie plus universelle de la réforme Castonguay-Nepveu. Le RAPC devient alors un obstacle qui freine la mise en oeuvre de la réforme au Québec au cours des années soixante-dix et au début des années quatre-vingt. 


\section{Un bilan québécois des quinze premières années du Régime d'assistance publique du Canada (1966-1981) La dimension sociale}

Yves VAILLANCOURT

Professeur et politicologue Département de travail social Université du Québec à Montréal

\section{INTRODUCTION ${ }^{1}$}

La littérature canadienne sur le Régime d'assistance publique du Canada (RAPC) met souvent en relief I'apport de ce programme fédéral de partage de coûts dans le développement des programmes de sécurité du revenu et de services sociaux dans diverses provinces canadiennes. Dans ces textes, le RAPC est fréquemment présenté comme un levier qui a joué un rôle fort positif dans le développement des programmes et des normes dans le champ de l'assistance publique, dans plusieurs provinces canadiennes, au cours des années 60 et 70 en particulier (Vaillancourt, 1991a)².

Dans le présent article, je compte démontrer que l'assertion rapportée ci-dessus mérite d'être nuancée profondément lorsqu'on la vérifie par rapport au cas du Québec. Dans le prolongement d'autres textes consacrés aux origines du RAPC (Vaillancourt, 1992a, 1992b), je compte dresser ici un 
bilan des quinze premières années de la partie québécoise de l'histoire du RAPC, soit la période allant de 1966 à $1981^{3}$. Pour découper davantage mon objet, je mettrai l'accent sur la dimension sociale plutôt que constitutionnelle du dossier, c'est-à-dire sur tout ce qui concerne l'influence du RAPC au Québec dans le développement des programmes de sécurité du revenu et de services sociaux ${ }^{4}$. En ce qui a trait à mes sources, je miserai principalement sur des données inédites tirées des Archives nationales du Canada et de quelques entrevues faites avec des informateurs clés ayant eu une expérience du RAPC au cours de la période considérée.

Le bilan que je présente sur l'influence du RAPC au Québec est construit à partir de l'hypothèse suivante : le RAPC, en raison de son orientation hautement sélective, s'est révélé un frein dans le développement des programmes de sécurité du revenu et de services sociaux québécois lorsque le Québec a voulu conférer une orientation plus universelle à ses programmes. Cette contradiction entre le RAPC et les programmes québécois est apparue moins vive dans les années 60 , sous le gouvernement de l'Union nationale, et plus vive dans les années 70 , sous les deux gouvernements de Bourassa et sous le premier gouvernement de Lévesque.

Afin de vérifier mon hypothèse, j'ai structuré mon texte en deux grandes parties. Dans la première partie, je m'emploie à dégager l'orientation sélective du RAPC en montrant comment les personnes pauvres constituaient la population cible de la législation de 1966 et le sont demeurées au cours de la période 1966-1981. Dans la deuxième partie, je présente un bilan du RAPC au Québec au cours de la période 1966-1981, en faisant ressortir comment cet instrument fédéral de partage de coûts a freiné la réforme Castonguay dans le champ sociosanitaire au cours des années 70 , dans la mesure où cette réforme touchait une clientèle plus universelle que celle visée par le RAPC.

\section{L'ORIENTATION SÉLECTIVE DU RAPC (1966-1981)}

Le RAPC est un programme fédéral de partage de coûts dont l'orientation, inscrite dans la législation de 1966, était hautement sélective. Cette orientation sélective a été maintenue par le gouvernement fédéral tout au long de la période considérée.

\section{La population cible du RAPC en 1966 : les pauvres}

Le RAPC, adopté en juillet 1966 (Canada, 1966), se présentait d'abord comme une bonification des anciennes lois fédérales de partage de coûts dans le domaine de l'assistance publique et, en particulier, comme une tentative d'élargir les possibilités de partage de la Loi sur l'assistance-chômage. 
Dans les derniers mois de négociations fédérales-provinciales menant au RAPC, grâce aux demandes insistantes de diverses provinces, dont le Québec, le devis initial avait été bonifié pour couvrir l'assistance aux mères nécessiteuses, certains "soins de santé », des "services de bien-être social », des services de " bien-être social à l'enfance », certains services administratifs, etc.

Toutefois, en dépit de ces initiatives de la onzième heure pour élargir la brèche concernant les « services de bien-être », le RAPC demeurait une loi de « dernier recours » conçue avant tout pour partager les coûts de l'aide sociale financière. Ainsi, sa matrice originale convenait mieux à l'aide sociale qu'aux services sociaux.

En somme, le RAPC se situait davantage en continuité qu'en rupture par rapport à son ancêtre, I'assistance-chômage. Il demeurait un « misnomer », tout comme l'assistance-chômage l'avait été avant lui, pour reprendre une épithète lancée un jour par Joe Willard. Ne l'oublions pas, I'aide " partageable » en vertu de l'assistance-chômage prenait la forme non seulement de chèques d'assistance versés à des personnes et à des familles touchées par le chômage, mais également celle de « services de bien-être » à des personnes qui étaient dans des institutions reconnues comme « établissements de soins spéciaux » et qui ne pouvaient pas travailler en raison soit de leur âge, soit d'un handicap, etc. Donc, bien avant le RAPC, I'assistance-chômage, en dépit de son nom trompeur, partageait des coûts de dépenses provinciales non seulement dans le domaine de la sécurité du revenu, mais également dans celui des services sociaux.

L'appellation RAPC était elle aussi un « misnomer », puisque les « services de bien-être » allaient toujours demeurer à l'étroit dans le concept $d^{\prime}$ " assistance publique», associé pour longtemps à l'aide sociale dans le subconscient de plusieurs. En conséquence, le RAPC serait enclin à traiter les services sociaux comme s'ils étaient des appendices de l'assistance sociale financière. L'orientation sélective du RAPC, normale dans le domaine de I'aide sociale, allait être imposée également aux services sociaux.

Pour mieux saisir le sens de mes assertions sur l'orientation sélective du RAPC, arrêtons-nous sur la clientèle cible visée par le RAPC, en partant d'abord de la législation de 1966.

Le RAPC n'est pas un programme social qui permet au gouvernement fédéral d'agir directement dans I'assistance publique. C'est plutôt un mécanisme financier qui permet au gouvernement fédéral de soutenir financièrement les provinces et les territoires, en partageant les coûts de l'assistance publique apportée sous diverses formes, soit par des établissements provinciaux, soit par des organismes municipaux, soit par des organismes non gouvernementaux [à but non lucratif] « approuvés par la province ». En vertu du RAPC, les provinces font parvenir au fédéral des réclamations pour des dépenses « partageables » engagées par des organismes dûment mandatés. 
Nous comprenons ici que la « partageabilité » des réclamations est définie à partir de paramètres inscrits dans la législation, la réglementation et les directives administratives fédérales et qu'il appartient à des fonctionnaires et à des vérificateurs fédéraux de s'assurer que les réclamations acheminées par les provinces sont recevables.

Parmi les règles qui déterminent la partageabilité des coûts, il y a d'abord celles inhérentes à quelques définitions placées dans l'article 2 de la loi (Canada, 1966).

L'assistance publique est définie comme celle qui s'adresse exclusivement aux " personnes nécessiteuses » (persons in need en anglais). Puis, de la manière propre à la tradition de la "Common $\operatorname{Law}^{5}$ ", huit formes de services d'assistance publique sont dûment mentionnées dans une énumération. Ces services peuvent avoir trait : a) aux " besoins fondamentaux » (nourriture, logement, vêtements, combustible, services d'utilité publique, fournitures ménagères) ; b) aux « besoins spéciaux » (articles, accessoires ou services) ; c) aux « soins dans un foyer de soins spéciaux » (autre qu'un hôpital, un établissement correctionnel ou « un établissement dont le principal objet est l'enseignement ») ; d) aux " déplacements et moyens de transport »; e) aux « obsèques et enterrements »; f) aux " services de soins sanitaires » ; g) aux « services prescrits de bien-être social dont l'acquisition est faite par un organisme approuvé par une province ${ }^{6} »$ ) aux " allocations de menues dépenses » pour des personnes hébergées par des institutions.

Le concept de personne nécessiteuse est défini de la façon suivante:

(a) une personne qui, par suite de son incapacité d'obtenir un emploi, de la perte de son principal soutien de famille, de sa maladie, de la perte de son invalidité, de son âge ou de toute autre cause acceptable pour l'autorité provinciale, est reconnue incapable (sur vérification par l'autorité provinciale qui tient compte des besoins matériels de cette personne et des revenus et ressources dont elle dispose pour satisfaire ces besoins) de subvenir convenablement à ses propres besoins ou à ses propres besoins et à ceux des personnes qui sont à sa charge ou de l'une ou l'autre d'entre elles, ou

(b) une personne âgée de moins de vingt et un ans qui est confiée aux soins ou à la garde d'une autorité chargée du bien-être social de l'enfance ou placée sous le contrôle ou la surveillance d'une autorité, ou une personne qui est un enfant placé en foyer nourricier selon la définition des règlements... (Canada, 1966 : art. 2).

Ainsi, la définition de " personne nécessiteuse » restreint le concept de besoins à un sens purement socio-économique ${ }^{7}$, sauf dans le cas des services de bien-être social à l'enfance qui sont traités de façon plus ouverte.

Mais plus loin, à l'occasion de la définition des "services de bien-être", une mince ouverture est faite. Ces services, à la différence d'autres compo- 
santes de l'assistance publique, ne sont pas destinés exclusivement aux « personnes nécessiteuses ». Ils peuvent être également destinés « à des personnes qui deviendront vraisemblablement des personnes nécessiteuses [...] si de tels services ne sont pas fournis »(Canada, 1966 : art. 2). Cette formulation offre la possibilité d'élargir la cible de l'assistance publique, de façon à admettre des personnes et des familles se situant « en proximité de besoins », pour utiliser une expression consacrée depuis.

Les services de bien-être social, à première vue, sont définis de façon assez large. Ils désignent :

les services qui ont pour objet d'atténuer, de supprimer ou de prévenir les causes et les effets de la pauvreté, du manque de soins à l'égard des enfants ou de la dépendance de l'assistance publique (Canada, 1966 : art. 2).

Cette définition générale est complétée par une liste de sept services particuliers qui semblent présentés dans le but de concrétiser la définition. En effet, il est spécifié que la liste est amenée « sans limiter la généralité de ce qui précède ${ }^{8} »$.

Donc, I'assistance publique partageable dans le cadre du RAPC vise des personnes qui ont passé un test de besoins permettant de faire la preuve qu'elles sont socio-économiquement nécessiteuses, ou susceptibles de l'être si elles n'avaient pas accès à certains services de bien-être. Ainsi le concept de besoin, dans le RAPC, est aplati à sa dimension financière. Les provinces peuvent toujours offrir des services d'assistance publique à une clientèle se situant au-dessus du seuil de pauvreté « harmonisable » avec les dispositions du RAPC, mais à condition de ne pas miser sur l'aide fédérale et d'en payer les frais à $100 \%$.

\section{Le RAPC change peu de 1966 à 1981}

Au fil des années, en dépit des nombreuses rumeurs qui par moments ont fait espérer le contraire, le RAPC a connu très peu de modifications sur le plan de son orientation fondamentale et de la population cible visée. Je distingue quatre phases dans l'évolution du RAPC au cours des années 1966 à 1981.

1) La première phase s'apparente à celle des débuts euphoriques du RAPC (1966-1970). C'est l'époque où les pionniers du RAPC à Santé et Bien-être Canada encouragent avec enthousiasme leurs vis-à-vis provinciaux à se prévaloir des possibilités du RAPC pour améliorer leurs programmes et développer de nouveaux services (Knight, 1990 ; Byrne, 1990 ; Rouleau, 1990). Cet aspect est même présenté comme un objectif du RAPC toujours mentionné dans les premiers rapports annuels : 
Le Régime a pour objectif de favoriser l'intégration, l'expansion et l'amélioration des régimes d'assistance publique provinciaux et municipaux, ainsi que l'amélioration et l'élargissement des services de bien-être social (MSNBES, 1968 : 1).

Dès la fin de l'année 1968 et le début de l'année 1969, la croissance des coûts devient préoccupante à Ottawa et dans plusieurs capitales provinciales, ce qui amène au début de 1969 la mise sur pied d'un comité fédéral-provincial pour étudier le problème (Willard, 1969b, 1969e). En outre, la direction de Santé et Bien-être incite les provinces à tenir compte plus rigoureusement de la ligne de démarcation entre le « correctionnel » et le « bien-être » en protection de la jeunesse ${ }^{9}$ et à éviter les interprétations trop libérales du concept de besoins ${ }^{10}$.

2) Au tout début des années 70 (1970-1972), dans un contexte marqué par les pressions des mouvements sociaux dans diverses régions du Canada en faveur de politiques gouvernementales plus efficaces dans la lutte contre la pauvreté, les limites du RAPC commencent à être critiquées à l'extérieur et à l'intérieur du gouvernement. La direction même de Santé et Bien-être semble gagnée à l'idée d'amender le RAPC pour qu'il puisse partager les coûts de programmes mis en œuvre pour soutenir les « pauvres qui travaillent ». Le gouvernement fédéral, au moment des discussions intenses sur les politiques sociales avec le Québec avant la Conférence de Victoria de juin 1971, envisage sérieusement la possibilité d'amender le RAPC, de façon que les provinces utilisant un test de revenus ${ }^{11}$ au lieu d'un test de besoins, dans des programmes de supplément de revenus et certains domaines de services sociaux, puissent avoir accès au partage des coûts dans le cadre du RAPC ${ }^{12}$. Ces hypothèses reviennent encore dans les discussions internes à Santé et Bien-être au cours de I'année 1972 (Pépin, 1972). Mais en dépit de ces délibérations, le RAPC n'est pas modifié au cours des années 1970 à 1972.

3) Pendant « la révision de la sécurité sociale »(1973-1978), plusieurs tiennent pour acquis que le RAPC est devenu un programme dépassé et condamné à disparaître bientôt au profit de nouveaux programmes plus adéquats. Durant cette fébrile période de discussions fédérales-provinciales, des comités de travail distincts préparent des esquisses de nouveaux programmes qui paraissent sur le point d'être adoptés. Il est ainsi question en 1975-1976 d'instaurer un nouveau programme de revenu minimum garanti à deux paliers, qui aurait pour effet de disposer de la composante sécurité du revenu du RAPC. Ce projet avorte en 1976. En 1977-1978, la priorité se déplace en direction du dossier des services sociaux. En 1977, la discussion se fait autour d'un projet de loi sur les services sociaux (le «Bill C-57 »), qui permettrait d'instaurer un nouveau programme à frais partagés exclusivement pour les services sociaux. Mais ce projet de loi est 
retiré pour être remplacé quelques mois plus tard, en 1978, par un autre, le projet de loi sur le financement des services sociaux (le « Bill C-55»), structuré cette fois à partir de la formule du financement global des programmes établis de 1977 (Canada, 1977). Mais ce projet est retiré très rapidement ${ }^{13}$.

Paradoxalement, pendant ces années où l'énergie fédérale-provinciale est consacrée à l'élaboration de belles esquisses qui demeureront sur le papier, plusieurs personnes, confondant leurs espoirs avec la réalité, avaient fini par oublier le RAPC qui pourtant était toujours là (Byrne, 1990). Au cours de ces six années, les provinces, parfois en pleine période de développement de nouveaux programmes sociaux comme au Québec, cessent de faire pression sur le RAPC pour obtenir des changements qui permettraient à ce mécanisme fédéral de partage de coûts de s'adapter aux exigences des temps nouveaux et de soutenir les nouvelles programmations provinciales. Au contraire, à force d'agir comme si le RAPC allait disparaître, plusieurs personnes d'Ottawa et d'ailleurs légitimèrent une sorte de moratoire concernant tout développement du RAPC. En ces années où les finances publiques fédérales étaient encore prospères, I'opération révision de la sécurité sociale devint un prétexte pour ne pas toucher au RAPC. Dans son entrevue, Ron Yzerman, un fonctionnaire fédéral expérimenté en ce qui touche le RAPC, a attiré mon attention sur les conséquences néfastes de l'oubli du RAPC au cours des années de la révision :

Let me describe something ! When this new act [Bill C-57 d'abord et Bill C-55 ensuite] was being talked about [...] there were a lot of curbs put on the CAP because we did not want to expand CAP. If CAP took over things the new act was going to do, why did we need a new act ? So not only were the resources such as the consultants stripped from the CAP Director, but there was an imposition that CAP should not develop and expand (Yzerman, $1988:$ 7-8).

Cette phase de non-développement et de non-expansion du RAPC en tant que programme fédéral était d'autant plus significative qu'elle contrastait, à la même époque, avec des phases de développement et d'expansion des programmes dans diverses provinces, comme nous le verrons ci-dessous en nous référant au cas du Québec.

4) Après la révision de la sécurité sociale qui se termine en queue de poisson en 1978, la transformation du RAPC continue d'être bloquée, à Ottawa, pour des raisons qui relèvent cette fois de la conjoncture économique, plutôt que politique. À partir de 1978, les finances publiques du gouvernement fédéral deviennent une source d'inquiétude et les mots d'ordre de rationalisation et de restriction budgétaire commencent à jeter leur ombre intimidante sur les programmes sociaux fédéraux dont le RAPC. 
Donc, pour le RAPC, l'heure est passée où il était encore possible d'entrevoir des changements qui auraient des répercussions financières. Dans un tel contexte, la population cible du RAPC ne peut plus être élargie. Elle doit demeurer restreinte aux socio-économiquement faibles ciblés dans la législatidemeure plus que jamais le programme sélectif qui avait été bâti dans les années 60.

\section{L'IMPACT DU RAPC SUR LES PROGRAMMES QUÉBÉCOIS (1966-1981)}

En prenant la réforme du système sociosanitaire québécois amenée par le " chapitre $48^{14}$ » en décembre 1971 comme moment charnière, je distingue trois sous-périodes, soit celle qui précède le chapitre 48 (1966-1970), ensuite celle de l'arrivée du chapitre 48 (1971) et, enfin, celle d'après le chapitre 48 (1972-1981). Mais avant de faire cette distinction, il est utile de se doter d'une

Sommes versées au Québec et dans l'ensemble des provinces canadiennes en vertu du RAPC de 1967-1968 à 1981-1982 (en millions de dollars)

\begin{tabular}{lccc}
\hline Année fiscale & Au Québec & Au Canada & Québec/Canada \\
$1967-1968$ & 117,1 & 342,7 & $34,2 \%$ \\
$1968-1969$ & 135,1 & 391,9 & $34,5 \%$ \\
$1969-1970$ & 156,6 & 450,1 & $34,8 \%$ \\
$1970-1971$ & 206,6 & 597,4 & $34,6 \%$ \\
$1971-1972$ & 271,5 & 727,4 & $37,3 \%$ \\
$1972-1973$ & 293,2 & 768,2 & $38,1 \%$ \\
$1973-1974$ & 318,5 & 824,8 & $38,6 \%$ \\
$1974-1975$ & 396,0 & 1056,1 & $37,5 \%$ \\
$1975-1976$ & 493,2 & 1379,5 & $35,8 \%$ \\
$1976-1977$ & 614,8 & 1602,4 & $38,4 \%$ \\
$1977-1978$ & 589,5 & 1561,8 & $37,7 \%$ \\
$1978-1979$ & 664,0 & 1683,8 & $39,4 \%$ \\
$1979-1980$ & 738,6 & 1894,9 & $39,0 \%$ \\
$1980-1981$ & 851,3 & 2205,8 & $38,6 \%$ \\
$1981-1982$ & 993,0 & 2612,0 & $38,0 \%$ \\
\hline
\end{tabular}


vue d'ensemble de l'impact du RAPC au Québec au cours de l'ensemble de la période.

Le Québec fut la dernière province canadienne à signer, en 1967, un accord avec le fédéral en vertu du RAPC. L'accord était rétroactif à partir de I'année 1966 (Québec et Canada, 1967). Le tableau qui suit porte sur I'évolution des dépenses effectuées au Québec et dans l'ensemble du Canada dans le cadre du RAPC au cours de la période 1967-1982.

Dès les premières années de mise en application du RAPC, il apparaît que le Québec accapare une part imposante des sommes versées dans I'ensemble du Canada. Ainsi, de 1967-1968 à 1970-1971, le Québec reçoit entre $34 \%$ et $35 \%$ des sommes versées dans le cadre du RAPC. Cette part augmentera à $37 \%$ et se maintiendra entre $37 \%$ et $39 \%$ au cours des années $70^{15}$.

La ventilation des sommes versées au Québec et dans I'ensemble du Canada en vertu du RAPC permet de constater que la part consacrée à I'assistance générale se situe à quelques points en dessous ou au-dessus de $60 \%$ au Québec et de $65 \%$ au Canada ${ }^{16}$.

\section{Avant le " chapitre 48 ", les programmes québécois s'harmonisent bien avec le RAPC (1966-1971)}

Quelques précisions et données factuelles peuvent aider à mesurer les retombées de l'accord au cours des années 60 .

Au moment de la signature de l'accord, en 1967, le Québec avait déjà quatre autres accords avec le fédéral dans le domaine de l'assistance publique, dont l'accord sur l'assistance-chômage en vigueur depuis 1959. L'accord sur le RAPC signifiait l'amorce d'une période de transition au cours de laquelle le RAPC serait amené graduellement à prendre la relève des quatre autres programmes en tant que mécanisme de partage des coûts dans le domaine de l'assistance ${ }^{17}$. Il faudra attendre une dizaine d'années pour que le RAPC devienne le seul programme fédéral à frais partagés dans I'assistance publique au Québec ${ }^{18}$. Ainsi, les sommes versées au Québec en vertu du RAPC représentaient 20,6\% des sommes versées pour les cinq accords dans le domaine de l'assistance en 1966-1967, $59 \%$ en 1967-1968, $83 \%$ en $1968-1969,87,3 \%$ en 1969-1970,92,9 \% en 1970-1971, 99,2 \% en 19711972 et $99,5 \%$ en $1972-1973^{19}$.

Pour mettre en perspective les chiffres et les faits présentés ci-dessus, il est utile de tenir compte de quelques traits du contexte politique et socioéconomique de la fin des années 60 et du début des années 70 qui ont eu un impact sur les politiques sociales en matière de sécurité du revenu et de services sociaux. 
1) Durant cette période, le taux de chômage est en augmentation au Québec et au Canada. Il se situe à $10 \%$ au Québec au tournant des années 60 et 70. Cette augmentation du chômage a un effet à la hausse sur les dépenses des programmes d'assistance sociale, ce qui provoque de l'inquiétude à Ottawa et dans d'autres capitales provinciales (Beausoleil, 1990).

2) On assiste au cours des années 1966 à 1971 à une radicalisation du mouvement syndical et à l'émergence de nouveaux organismes communautaires, appelés souvent " comités de citoyens », qui prennent en charge la défense des droits des chômeurs et des assistés sociaux. Les organisations syndicales et communautaires s'unissent pour revendiquer des gouvernements, à Québec comme à Ottawa, une reconnaissance du droit à l'aide sociale et à des prestations décentes. Elles réclament aussi plus de démocratie dans la planification et l'administration des programmes d'assistance publique. Ces mobilisations populaires jouent un rôle important au moment des assises de la Commission parlementaire sur l'aide sociale à Québec en 1969 et du Comité sénatorial sur la pauvreté à Ottawa en 1970 (Cloutier, 1991 ; Escojido, 1991).

3) Au cours des années 1966 à 1970, le gouvernement de l'Union nationale est plus timide et conservateur que le gouvernement précédent en matière de politiques sociales ${ }^{20}$. Ce conservatisme social imprimé à partir du cabinet a eu pour effet de ralentir les démarches entreprises sous les gouvernements précédents pour instaurer des réformes dans le domaine de l'aide sociale et de l'assurance-maladie. Pour l'assurance-maladie, le conservatisme de I'Union nationale a signifié une préférence pour une approche sélective du medicare comme celle qu'on trouve aux ÉtatsUnis $^{21}$.

4) En dépit du penchant pour le conservatisme social du gouvernement de I'Union nationale (UN), le ministère de la Famille et du Bien-être social, sous Jean-Paul Cloutier, a quand même poursuivi jusqu'à un certain point I'action entreprise dans certains dossiers de politiques sociales sous René Lévesque, le ministre précédent ${ }^{22}$. Contrairement à d'autres de ses collègues au cabinet, Cloutier n'était pas prisonnier de la politique partisane. Comptable de formation, il avait du respect pour l'apport des professionnels et des fonctionnaires compétents. II s'empressa de faire confiance à l'expertise et aux propositions de l'équipe mise en place au temps de Lévesque ${ }^{23}$. Pendant ces années, le Ministère augmenta son soutien financier aux agences de service social, tout en mettant de l'ordre dans les règles administratives à suivre pour obtenir cette aide ${ }^{24}$. II continua à développer un réseau de bureaux locaux et régionaux pour la distribution de I'assistance-chômage et à inviter les agences à se délester de l'administration de l'aide sociale, afin de mieux se concentrer sur les services 
sociaux (Marier, 1969, 1990a, 1990b ; MFBES, 1970). Il s'occupa de la préparation des multiples versions de la nouvelle législation sur l'aide sociale et de la réglementation connexe. La nouvelle loi fut adoptée à I'automne 1969, mais sa mise en application ne devait commencer qu'en novembre 1970 sous un nouveau gouvernement (Québec, 1969 ;MFBES, 1970).

5) Pour les dossiers qui nous intéressent, soit ceux qui ont des interfaces avec le RAPC, la conclusion à dégager de ce qui précède est la suivante : sous le gouvernement de I'Union nationale, en dépit de l'apport de l'équipe dirigeante au ministère de la Famille et du Bien-être, la réorganisation de I'aide sociale et des services sociaux a progressé à pas de tortue. Ainsi la réforme de l'aide sociale, recommandée par le rapport Boucher et relancée par René Lévesque en 1965-1966, a traîné de façon interminable entre 1966 et l'automne de 1969, en raison principalement des réactions conservatrices de membres du cabinet, dont Paul Dozois, l'influent ministre des Finances, devant les multiples devis de projets de loi préparés par le Ministère ${ }^{25}$. Quant à la réforme des services sociaux, elle progressait dans certains secteurs, dont celui de la jeunesse, mais ces avancées sectorielles étaient compromises par le maintien au cours des années 60 du carcan de la vieille loi québécoise de I'assistance publique (Québec, 1964) dont I'esprit demeurait incroyablement désuet et qui ne serait pas abrogée avant I'adoption du chapitre 48 (Québec, 1971).

Pour ce qui concerne plus spécifiquement l'administration de l'accord du RAPC au Québec, au cours de ces années, l'harmonisation entre le programme fédéral et les programmes québécois ne causait pas de problème majeur.

Par ailleurs, dans le domaine de l'assistance générale, les lenteurs entourant la réforme de l'aide sociale posaient des problèmes aux fonctionnaires fédéraux à la fin des années 60, puisque la philosophie de la nouvelle loi d'aide sociale au Québec était faite sur mesure, plus que celle des vieilles lois toujours en vigueur, pour bien s'harmoniser avec celle du RAPC ${ }^{26}$. Mais comme les anciennes lois fédérales de partage de coûts demeuraient dans le décor, les réclamations qui heurtaient les dispositions du RAPC pouvaient toujours être canalisées vers d'autres programmes fédéraux à frais partagés.

Quant aux programmes québécois de services sociaux, leur caractère sélectif en faveur des plus pauvres leur permettait de satisfaire aisément les exigences du RAPC, en ces années antérieures à la réforme Castonguay. Le fait qu'un grand nombre d'agences de service social s'occupaient encore de l'administration de l'aide sociale, ou avaient conservé une programmation marquée par les habitudes prises à l'époque où elles s'occupaient de l'aide sociale, se trouve paradoxalement à conférer aux agences une image qui les rend plus admissibles au partage des coûts des services sociaux. Ainsi, 
I'identification des agences à une clientèle de personnes à faibles revenus est un atout pour répondre aux critères sélectifs du RAPC. Donc, le Québec n'a pas de difficulté particulière à faire accepter par le fédéral ses réclamations pour le partage des coûts des services sociaux ${ }^{27}$.

\section{Au début des années 70, quatre réformes, dont le " chapitre 48 ", ont des incidences sur l'administration du RAPC}

Au début du premier mandat du gouvernement Bourassa, avec Claude Castonguay à la tête du nouveau ministère des Affaires sociales (MAS), quatre réformes ayant des interfaces avec le RAPC sont à l'agenda du nouveau gouvernement.

La première réforme, celle de l'assurance-maladie à partir de novembre 1970, ne cause pas de problème particulier, parce que la loi québécoise à cet effet (Québec, 1970) s'inscrit très bien dans la logique universelle de la loi fédérale sur les services médicaux de 1966. L'arrivée de l'assurance-maladie universelle au Québec a toutefois pour conséquence d'absorber les coûts de I'assistance médicale instaurée en avril 1966 et partagée par le RAPC depuis ${ }^{28}$.

La deuxième réforme est celle de l'aide sociale, également mise en route en novembre 1970. Cette réforme s'inscrit bien à l'intérieur des paramètres du RAPC, puisque sa philosophie de base est axée sur le concept de besoins, tout comme celle du RAPC ${ }^{29}$. L'entrée en vigueur de la nouvelle loi d'aide sociale au Québec amène des augmentations de coûts élevées en 1970-1971 et 1971-1972, comme en témoignent les chiffres du tableau de la page $26^{30}$; mais ces coûts entraînés par un programme d'aide sociale globale concernant des personnes et des familles à faibles revenus étaient clairement partageables en vertu du RAPC.

La troisième réforme correspond au projet d'instaurer un nouveau programme de revenu minimum garanti (RMG), qui était une priorité de Castonguay au cours des années 1971 et 1972, même s'il ne s'est pas concrétisé pour des raisons ayant trait à la dimension constitutionnelle (Vaillancourt, 1991c). Ce projet de RMG porté par Castonguay était issu des recommandations du volume V du Rapport de la Commission CastonguayNepveu, paru au tout début de 1971 (Castonguay et Nepveu, 1971a, 1971b). Avec les allocations familiales profondément restructurées, le RMG représentait l'une des deux pièces centrales de la "politique sociale intégrée » de Castonguay. Ces propositions avaient semé une profonde inquiétude dans le camp fédéral à la veille de la Conférence de Victoria de juin 1971. Elles avaient jeté une ombre sur les propositions du Livre blanc de Munro (1970) rendue publiques à la fin de 1970.

Ce qu'il faut principalement mettre en lumière ici, c'est que les propositions de Castonguay concernant le RMG supposaient une nouvelle réforme 
de I'aide sociale au Québec. Cette réforme, sur le plan du financement, exigeait une harmonisation délicate avec le RAPC. En effet, l'instauration d'un programme de RMG au Québec n'aurait pas pu être partageable à l'intérieur du RAPC, puisque le RMG aurait été basé sur un test de revenus, alors que le RAPC exigeait un test de besoins et de ressources. Dans les discussions Québec-Ottawa qui deviennent fort tendues en 1971 et 1972, la question des interfaces entre le RMG québécois et le RAPC fédéral constitue une importante pierre d'achoppement pour le camp fédéral. De son côté, le camp québécois était surpris par les difficultés rencontrées, parce que le MAS, tout comme la Commission Castonguay-Nepveu avant lui, dans l'élaboration de ses propositions sur la politique sociale intégrée, n'avait tenu compte que superficiellement des contraintes propres au fédéralisme fiscal. De toute manière, la campagne menée par Castonguay en 1971 et 1972 pour une "politique sociale intégrée » s'est enlisée, avant que les réformes proposées puissent être mises en œuvre. Une impasse s'est développée et un étonnant dénouement est survenu au début de l'année 1973, à la suite de l'arrivée de Marc Lalonde à Santé et Bien-être Canada et de la publication de son Livre orange (Lalonde, 1973) ${ }^{31}$.

Ainsi, en raison de certaines limites du RAPC, le troisième projet de réforme de Castonguay resta dans les cartons, avant d'être ressorti quelques années plus tard ${ }^{32}$.

La quatrième réforme est amenée par le « chapitre 48 » concernant la réorganisation des services de santé et des services sociaux (Québec, 1971). Cette réforme, adoptée en décembre 1971, comportait l'intégration des services de santé et des services sociaux à l'intérieur d'un même système et la poursuite d'une certaine universalité. Un des objectifs de la réforme était formulé comme suit :

[...] rendre accessible à toute personne, d'une façon continue et pendant toute sa vie, la gamme complète des services de santé et des services sociaux, y compris la prévention et la réadaptation, de façon à répondre aux besoins des individus, des familles et des groupes aux plans physique, psychique et social (Québec, 1971 : art. 3 (b) ).

Ce libellé supposait que les services sociaux autant que les services de santé devaient être destinés " à toute personne », peu importe la situation de ses revenus. En d'autres termes, la population cible du système sociosanitaire, à la différence de celle du RAPC, ne correspondait pas seulement aux personnes socio-économiquement faibles sur le plan financier. L'orientation universelle du chapitre 48 ne posait pas de problèmes considérables pour les services de santé, dans la mesure où la législation fédérale correspondante, soit celle portant sur l'assurance-hospitalisation et le medicare, était elle-même caractérisée par une orientation universelle. Mais dans le cas de la composante des services sociaux, la nouvelle législation et la réforme qui en décou- 
lerait entraient en contradiction avec la législation fédérale correspondante, soit le RAPC dont I'orientation était sélective.

\section{La contradiction entre I'universalité $d u$ " chapitre 48 » et la sélectivité du RAPC}

Cette contradiction entre le chapitre 48 et le RAPC devait soulever un certain nombre de problèmes liés au partage fédéral-provincial des coûts des programmes de services sociaux québécois. Elle a été bien cernée par plusieurs gestionnaires de l'époque, tant à Québec qu'à Ottawa. Jacques Brunet, le sous-ministre en titre, en était fort conscient :

[...] Pour nous il était important que les services sociaux puissent devenir plus universels. On a fait, dès le départ, en 1970, 1971, 1972 , une distinction très nette entre ce qu'on appelait, nous autres, le contenu des services sociaux et la pauvreté ou l'insuffisance de revenus. On a fait beaucoup d'efforts pour que ce soit vu comme deux choses distinctes et pour que les services sociaux deviennent plus universels, plus accessibles à tout le monde en fonction des besoins. Nous ne voulions pas faire des services sociaux juste une question de services pour les pauvres. C'était aussi une façon de revaloriser, dans notre esprit, les services sociaux, de s'assurer qu'ils étaient accessibles en fonction des besoins et pas juste en fonction d'un critère financier [...] Évidemment, la loi du RAPC ne nous aidait pas (Brunet, $1990: 6$ ).

Voici comment Aubert Ouellet, un sous-ministre adjoint au MAS très influent dans les négociations fédérales-provinciales au cours des années 70, résume ce qui s'est passé à la suite de l'adoption du chapitre 48 :

[...] lorsque le gouvernement du Québec a établi le cadre politique et le cadre législatif à l'intérieur duquel devaient se donner les services sociaux, il n'a pas tenu compte de la contrainte du RAPC. [...]. On voit bien dans cette loi-là [le chap. 48] que les services sociaux sont considérés comme un droit pour l'ensemble de la population québécoise, sans considération de ressources. Comme le gouvernement fédéral n'a pas adapté sa législation, nous avons tenté de manœuvrer à l'intérieur des contraintes qui nous étaient imposées et nous avons essayé de faire en sorte que tous les services dispensés à des personnes défavorisées sur le plan socio-économique soient partageables par le gouvernement fédéral. Cela laissait évidemment en plan les services distribués à des personnes non défavorisées sur le plan socio-économique, puisque ces services n'étaient pas financièrement partageables par le gouvernement du Canada. C'est ainsi que le Québec paye une partie des services, seul. Et ça dure toujours depuis ce temps-là (Ouellet, 1989 : 3-4). 
À ces témoignages de fonctionnaires québécois, il est intéressant d'en ajouter un autre, pas moins lucide, d'un fonctionnaire fédéral. Invité à commenter mon hypothèse concernant la contradiction entre le RAPC d'orientation plutôt sélective et le chapitre 48 d'orientation plutôt universelle, Ron Yzerman a répondu :

Absolutely! That is exactly what took place. There is no question that, in my mind, the direction was towards universality or a social utility concept. And because CAP is a selective cost sharing instrument and is designed to support programs that are really targeted, [the end result] is that the burden that provinces had to assume to comply with CAP requirements, in order to secure the maximum cost sharing for their programs, increased in the province. It did certainly [impact] in our discussions with our provincial counterparts in Quebec. They had a good deal of difficulty in understanding our limitations and feeling that to a degree we were unreasonable or perhaps, more correctly, that CAP was demonstrating its inflexibility ; and that it was becoming more and more obvious that, as an instrument, as a social policy instrument if you will, it was not keeping up with the time (Yzerman, $1988: 7$ ).

Ces remarques d'Yzerman m'apparaissent fort éclairantes, surtout si on les relie à celles rapportées antérieurement concernant le non-développement du RAPC pendant la révision de la sécurité sociale de 1973 à 1978. Tandis que le fédéral refusait de modifier le RAPC, une dynamique intense et progressiste de développement social avait cours dans certaines provinces, dont le Québec. À ce moment-là, la contradiction relevée plus haut ne pouvait que faire mal.

\section{Après le " chapitre 48 ", les programmes québécois s'harmonisent moins bien avec le RAPC (1972-1981)}

Même si la planification en avait été amorcée au cours des années 60, la Révolution tranquille dans le domaine des services sociaux eut lieu seulement dans les années 70 . Elle fut mise en route sous le gouvernement Bourassa et s'intensifia sous le premier mandat du gouvernement péquiste, soit au moment où Denis Lazure, ministre social-démocrate, dirigeait le MAS dans une conjoncture où les finances publiques devenaient serrées. Ainsi, les années 70 sont pour les services sociaux, sous le PLQ et le PQ, des années de réforme, de modernisation et de développement. La demande sociale issue des mouvements sociaux demeure soutenue et incite le gouvernement à impulser des réformes.

Il y aurait des différences à faire ressortir concernant la politique sociale du gouvernement Bourassa et celle du gouvernement Lévesque au cours des années 70. Mais attendu l'objet et l'hypothèse privilégiés dans le présent 
article, je mets l'accent sur l'aspect de la continuité entre ces gouvernements. Dans le domaine des services sociaux, au cours des années 1972 à 1981, sous Bourassa comme sous Lévesque, le gouvernement du Québec déploie des efforts significatifs pour réaliser la réforme et poursuivre les grands objectifs du chapitre 48. Cela veut dire que plusieurs des initiatives gouvernementales dans le domaine social visent une clientèle plus large que celle admissible dans le RAPC pour le partage des coûts à 50/50 avec les provinces.

À ses origines, le chapitre 48 n'avait pas été conçu par des personnes soucieuses de vérifier à tout instant la partageabilité des coûts à l'intérieur du RAPC. Au Québec, à la différence des réflexes établis dans d'autres provinces, la question de l'arrimage de la réforme au RAPC était posée à la fin et non pas au début des exercices de planification sociale ${ }^{33}$.

Mais lorsque la réforme du système sociosanitaire a commencé à être mise en route avec détermination, à partir des années 1972-1973, des zones de friction ne tardèrent pas à surgir dans les négociations Québec-Canada sur la partageabilité des coûts. L'apparition par exemple de nouveaux établissements dans le réseau des affaires sociales, comme les Conseils régionaux de la santé et des services sociaux (CRSSS), les Centres de services sociaux (CSS), les Centres locaux de services communautaires (CLSC), etc., posait de nouveaux problèmes aux fonctionnaires fédéraux responsables de l'administration du RAPC. Sans être propres au Québec, ces problèmes avaient une intensité plus grande que dans les autres provinces.

Ainsi comment distinguer, au MAS d'abord, puis dans les CRSSS et les CLSC, la composante sociale de la composante santé des services et des coûts $^{34}$ ? Puis, dans les services sociaux, quelle partie des activités s'adressait à une clientèle cible du type de celle qui était reconnue par le RAPC ?

De même, dans les CSS, quels services étaient partageables en vertu des dispositions du RAPC ? Comment soustraire les coûts des services sociaux donnés en milieu scolaire ou hospitalier qui sont non recevables à I'intérieur du RAPC ? ${ }^{35}$

Ensuite, dans les centres d'accueil pour jeunes, comment distinguer la partie des coûts et des services qui appartient vraiment au domaine des « services de bien-être à l'enfance » de celle qui relève du domaine correctionnel ou de la justice, qui est non partageable en vertu du RAPC ?

Les négociations ayant trait au partage des services sociaux donnés en CSS furent particulièrement difficiles au cours des années 1974 et 1975. À la différence des agences de service social, les CSS ne semblaient pas offrir des services aux seules personnes dans le besoin financier. Il y avait des adoptions faites dans des familles de classes moyennes. Il y avait des cas de délinquance dans des familles à revenus moyens, voire élevés. Comment départager tout cela, sans être obligé de faire passer un test de besoins et de ressources à chaque personne appartenant à la clientèle des CSS ? Avec le temps, on a fini 
par s'entendre sur une procédure par échantillonnage. En bout de ligne, la décision a été prise de reconnaître 90 \% de la clientèle des CSS comme recevant des services partageables en vertu du RAPC. Quant aux coûts pour les autres 10 \% de la clientèle, le Québec devait les payer seul (Duperré, 1988 ; Ouellet, 1988 ; Bédard, 1990 : 6 ; Byrne, 1990 : 19).

Ces problèmes qui ont perduré dans les négociations Québec-Canada pour le partage des coûts en vertu du RAPC tout au long des années 70 étaient à la source de nombreuses lourdeurs bureaucratiques ${ }^{36}$. Dans plusieurs dossiers, les négociations pouvaient s'étendre sur des années. «Ça finissait pas [...] Le fédéral était juge et partie. Il avait le gros bout du bâton », pour reprendre l'expression d'André Bédard, un fonctionnaire qui négociait pour le Québec les réclamations reliées au RAPC dans les années 70 (Bédard, 1990 : 5-6).

Sans dresser une liste complète, voici quelques exemples de développement de nouveaux services qui faisaient apparaître de nouvelles zones de tension entre Québec et Ottawa pour le partage des coûts. II y avait, notamment, la création des Bureaux d'aide juridique dans le sillage de la législation de 1972 ; la politique des services de garde à l'enfance, mise en branle sous Bourassa en 1974 (le «Plan Bacon ») et intensifiée sous Lévesque-Lazure avec I'adoption d'une nouvelle loi et la création de l'Office des services de garde (Québec, 1979) ${ }^{37}$; I'adoption d'une nouvelle loi concernant les services aux personnes handicapées et permettant la création d'un Office pour ces mêmes personnes en 1978 (Québec, 1978) ; I'intensification de la politique de maintien à domicile en 1979 ; I'instauration d'une nouvelle politique de protection de la jeunesse dans la foulée de l'adoption d'une nouvelle loi en 1977 (Québec, 1977) ; la politique de soutien gouvernemental à de nouvelles ressources communautaires pour les femmes victimes de violence et pour les jeunes à partir de la fin des années 70 .

Dans ces champs d'action sociale et d'autres analogues, des problèmes surgissent relativement à la question du financement, parce que le gouvernement du Québec, au cours des années 70, vise l'instauration de services qui, sans être pleinement universels, se trouvent néanmoins à être sélectifs et plus universels que ceux qui sont reconnus dans la tradition du RAPC ${ }^{38}$. Dans les garderies, par exemple, un problème persistait depuis 1975, comme l'a rappelé André Bédard : «Parce que le programme québécois [de services de garde], par rapport au RAPC, avait un excès de générosité, le fédéral ne partageait pas »(Bédard, $1990: 4)^{39}$.

\section{CONCLUSION}

Aussi longtemps que les provinces canadiennes ont voulu utiliser le RAPC pour réformer leurs programmes $d^{\prime}$ aide sociale en visant une plus grande 
accessibilité ou encore pour réorganiser leurs services sociaux en ciblant une clientèle composée de personnes socio-économiquement démunies prêtes à se plier à un test de ressources et de besoins, elles ont pu apprécier les virtualités du RAPC comme instrument fédéral de partage de coûts. C'est ce qui s'est passé dans diverses provinces canadiennes, y compris au Québec, au cours des années 60.

Les limites du RAPC ont commencé à apparaître au tournant des années 60 et 70, lorsque certains gouvernements provinciaux aux orientations sociales plus progressistes ont voulu mettre de l'avant des initiatives, des idées qui se butaient au mur des règles du jeu sélectives du RAPC. Le gouvernement fédéral ne pouvait plus, à partir d'un instrument de partage comme le RAPC, qui ne " bougeait " pas, répondre aux nouvelles demandes de provinces qui, comme le Québec, « bougeaient » sur le plan des politiques sociales. Comme le disait en entrevue Thomas Duperré, un fonctionnaire québécois engagé dans la négociation de plusieurs dossiers délicats des années 70 touchant le RAPC :

Quand le fédéral se trouve placé devant une nouvelle approche pour lutter dans les provinces contre la pauvreté, ça ne passe pas. Aussitôt qu'on veut sortir de la routine traditionnelle de l'aide sociale, ça devient très compliqué (Duperré, 1988 : 12).

C'est exactement ce qui est arrivé dans les tractations entre le Québec et le fédéral concernant le RAPC, au cours des années 70 . Chaque fois que le Québec arrivait avec des réclamations pour des programmes qui visaient une clientèle plus large que celle hautement sélective admise par le RAPC, les problèmes commençaient à surgir. Ces difficultés ont été vécues pour le partage des coûts des services de garde, des services aux personnes handicapées, du programme de Supplément au revenu de travail ou SUPRET, etc. Il n'était pas possible d'utiliser le RAPC pour partager les coûts de programmes ou de services qui ne ciblaient pas des personnes pauvres. Il n'était pas possible non plus d'interpréter de façon généreuse la clause de la « proximité de besoins », qui avait laissé entrevoir certains espoirs pour les services de bienêtre en 1966, mais qui a toujours été utilisée parcimonieusement par la suite.

Loin d'être un levier pour le développement des programmes provinciaux, le RAPC devenait un frein. Il s'est comporté, au cours des années 70, comme une camisole de force qui bloquait le développement d'une approche progressiste dans le champ des services sociaux, en refusant de partager les coûts engagés dans certaines provinces pour des services destinés par exemple aux classes moyennes de la société, ou encore à des pauvres qui travaillaient. Les provinces qui, comme le Manitoba, la Saskatchewan et la Colombie-Britannique, ont été dirigées par des gouvernements social-démocrates pendant une partie ou la totalité des années 70 ont vécu le même problème. Elles ne pouvaient pas être accompagnées et soutenues par le RAPC 
dès qu'elles décidaient d'offrir des services d'assistance publique à des couches de la population qui n'étaient pas totalement démunies sur le plan socioéconomique ${ }^{40}$.

En somme, tout au long des années 70, le RAPC, demeuré inchangé depuis son apparition en 1966, s'est trouvé soudainement dépassé par les expériences et les demandes sociales de certaines provinces qui voulaient aller de l'avant en construisant des services sociaux moins sélectifs. Au Québec, en ces années où la priorité des gouvernements Bourassa et Lévesque est conférée à la réalisation de la réforme Castonguay-Nepveu dans le domaine sociosanitaire, le RAPC devenait " un empêcheur de tourner en rond ». À partir de la fenêtre du RAPC, les objectifs mêmes du chapitre 48 avaient un air suspect. L'idée même de miser sur des établissements comme les CLSC et les CRSSS devenait une source de complication.

Certes, les négociateurs fédéraux et québécois parvenaient souvent à trouver des compromis et à déjouer certaines contraintes propres au RAPC. Les chiffres du tableau de la page 26 ont révélé que, des années 60 aux années 70, la part du Québec a même augmenté, passant de 34 \%-35 \% à $37 \%-38 \%$ de l'ensemble des sommes distribuées dans le cadre du RAPC au Canada. Cela montre que les efforts déployés par le Québec dans les années 70 pour moderniser et développer ses programmes ont bénéficié d'un soutien financier accru de la part du gouvernement fédéral. Mais cet accroissement quantitatif ne doit pas occulter une limite : le RAPC s'est souvent situé en travers des projets québécois, parce que leur approche n'était pas assez sélective à son goût.

\section{Notes}

1. Je remercie Mme Jaye Jarvis, archiviste, qui m'a guidé avec compétence, empressement et humour dans mes démarches de recherche aux Archives nationales à Ottawa en 1990 et 1991.

2. Comme le RAPC, en dépit de son importance, demeure encore trop peu connu au Québec, je dois rappeler aux lecteurs que ce programme fédéral à frais partagés concerne non seulement le financement du programme québécois d'aide sociale, mais également celui de dizaines de programmes de services sociaux reconnus comme "services de bien-être » dans la terminologie fédérale. Le RAPC entretient des interfaces avec la protection de la jeunesse, le placement en familles d'accueil, le soutien à domicile, I'aide juridique, les services aux personnes handicapées, les services de garde à l'enfance, I'hébergement des femmes victimes de violence, etc. Dans les budgets du MSSS, des Conseils régionaux, des CLSC, des CSS, de la Commission des affaires sociales, d'organismes non gouvernementaux, etc., une partie des dépenses sont " partageables » en vertu du RAPC. Malheureusement, au Québec, un nombre étonnant de gestionnaires, d'intervenants, d'étudiants, de professeurs, de chercheurs 
et même de ministres dans le champ du Bien-être sont peu au courant de ces faits. Cette lacune affaiblit nombre d'opinions, d'enseignements et de bilans dans le domaine de la sécurité du revenu, de la santé et des services sociaux au Québec.

3. Lorsque je fais référence au RAPC dans ce texte, je renvoie à la partie I sur l'assistance générale et les services de bien-être social, qui représente de loin la composante principale. Je ne me reporte pas à la partie II (art. 10-13) sur le " bien-être social des Indiens », qui n'a jamais été utilisée, ni à la partie III (art. 14-17) sur les « projets d'adaptation au travail », qui a été condamnée à un rôle marginal dès la naissance du RAPC, comme je l'ai montré ailleurs (Vaillancourt, 1992b).

4. Dans un autre article portant sur la même période, j'ai fait un bilan québécois du RAPC en mettant I'accent cette fois sur la dimension constitutionnelle (Vaillancourt, 1991c).

5. Dans la tradition de la « Common Law », à la différence de celle du Code civil, les éléments mentionnés dans une énumération servent à fermer les portes plutôt qu'à les ouvrir.

6. Dans la définition de l'assistance publique donnée à l'article 2 , les « services de bien-être social » apparaissent comme l'une des huit formes $d^{\prime}$ " assistance publique ». Par contre, dans les articles 4 et 5 consacrés aux accords entre le Canada et les provinces en vertu du RAPC, une distinction est faite entre les paiements versés au titre de l'assistance publique et ceux versés au titre des services de bien-être social, comme si ces services se situaient à l'extérieur de l'assistance publique. Cette contradiction entre la terminologie de l'article 2 et celle des articles 4 et 5 est significative d'un certain flou dans la définition des concepts et révèle que les artisans de la législation hésitaient entre la décision d'inclure la composante « services de bien-être social » dans le concept d'assistance publique, souvent traité en synonyme de celui de l'assistance sociale financière, ou de l'en exclure. En somme, I'appellation même du RAPC est un indice et une source de confusion.

7. Notons au passage que les « pauvres qui travaillent » sont exclus de la définition des « personnes nécessiteuses », parce que leur insuffisance de revenus n'est pas causée par la perte d'un emploi.

8. Ici encore, la tradition de la «Common Law » aura plus de poids que la garantie pourtant formellement offerte : au fil des ans, l'énumération permettra de fermer des portes au lieu d'en ouvrir.

9. Ce problème deviendra un irritant dès 1968 dans les négociations entre Québec et Ottawa au sujet du RAPC (Munro, 1968 ; Willard, 1969a, 1969c, 1969d). Ce contentieux persistera dans les années 70 et 80 (Cragg, 1972 ; Duperré, 1976, 1988, 1990 ; Bédard, 1990 ; Yzerman, 1988).

10. Au printemps de 1969, Willard envoie une lettre aux sous-ministres du Bien-être dans les provinces pour les inviter à modérer les interprétations trop libérales du concept de besoins : "The massive extension of expenditures at both levels of government since the Canada Assistance Plan Agreements were signed is evidence of the result. We are, however, becoming concerned by indications that some provinces, particularly in extending their health care, institutional care and welfare services programs may be moving beyond the point where it can be said that a test of need, within the intent of the Canada Assistance Plan, is being applied» (Willard, 1969h). 
11. Popularisé avec le programme fédéral de Supplément de revenus garantis pour les personnes âgées de 1967, le « test de revenus » est plus simple à administrer, plus libéral et moins "stigmatisant » que le « test de besoins » qui est en fait un " test de besoins et de ressources ». Le test de revenus s'apparente à un rapport d'impôt fait à partir d'un formulaire simple. Ce formulaire est habituellement rempli par la personne requérante elle-même qui l'utilise pour faire une déclaration concernant l'état de ses revenus au cours d'une période de référence. Si les revenus déclarés sont en dessous d'un certain plafond, la personne requérante a droit aux prestations en totalité ou en partie. Dans le cas du test de besoins, le formulaire est plus complexe et il est rempli, habituellement, par un fonctionnaire qui vérifie, à partir d'une enquête, s'il y a un écart entre les revenus et les biens de la personne requérante et ses besoins fondamentaux.

12. À la fin des années 60 et au début des années 70 , la direction du ministère de la Santé et du Bien-être social reprenait elle-même à son compte certaines hypothèses de réforme du RAPC qui auraient permis notamment de partager les coûts de programmes concernant les « pauvres qui travaillent » (Willard, 1969i, 1969k ; Munro, 1970, 1971a, 1971b, 1971c, 1971d, 1971e ; Carter, 1971 ; Leach, 1971 ; Préfontaine, 1971). Mais ces modifications n'eurent pas lieu. Puis, au cours de la « révision de la sécurité sociale » (1973-1978) déclenchée à la suite de la publication du Livre orange de Lalonde (1973), plusieurs tenaient pour acquise l'idée que le RAPC serait amendé substantiellement après I'adoption d'un programme de revenu minimum garanti et d'une nouvelle loi qui aurait régi le partage des coûts des services sociaux. Mais la révision se termina en queue de poisson et le RAPC, tel un miraculé, sortit indemne de ces cinq années d'intenses discussions fédérales-provinciales (CBNES, 1978 ; Canada, 1981 ; Duperré, 1976, 1987, 1988 ; Brunet, 1990 ; Ouellet, 1988 ; Osborne, 1986).

13. Sur les discussions fédérales-provinciales entourant les projets de loi C-57 d'abord et C-55 ensuite, voir CNBES, 1978 ; Duperré, 1987 ; Osborne, 1986 ; Canada, 1981 ; Vaillancourt, 1991c).

14. L'expression " chapitre 48 » dans cet article est utilisée pour désigner le chapitre 48 des Lois du Québec de l'année 1971, c'est-à-dire la Loi sur les services de santé et les services sociaux de décembre 1971. Il s'agit d'une expression consacrée par le sens commun dans le réseau des affaires sociales.

15. L'importance considérable des sommes reçues par le Québec dans le cadre du RAPC au cours des années 70 (38\% des sommes totales versées en vertu du RAPC au Canada) s'expliquent par quatre facteurs : le taux de chômage élevé, l'augmentation de I'accessibilité et des barèmes de l'aide sociale à la suite de la réforme de novembre 1970, l'importance conférée à l'institutionnalisation dans la stratégie de soutien aux personnes en perte d'autonomie (ce qui a un impact sur les dépenses des "foyers de soins spéciaux », beaucoup plus élevées au Québec qu'ailleurs) et l'orientation relativement progressiste des programmes de services sociaux développés sous les gouvernements des années 70 . A partir des années 80, la part du Québec dans le RAPC baissera, se situant autour de $31 \%$ au début des années 90 .

16. Calculs recueillis ou effectués à partir des Rapports annuels du Régime d'assistance publique du Canada des années 1968-1969 à 1981-1982 (MSNBES, 1968 à 1982).

17. Évidemment, au cours de ces années de transition, les vérificateurs fédéraux surveillaient les dédoublements. Les mêmes coûts d'assistance ne pouvaient pas être partagés deux fois en vertu de deux accords différents. 
18. Des sommes furent versées au Québec en vertu de l'accord sur l'assistancechômage jusqu'au milieu des années 70 . À la fin, ces sommes étaient rendues à quelques milliers de dollars seulement.

19. Calculs effectués par l'auteur à partir des chiffres du ministère fédéral des Finances de mars 1973 (Garner, 1973).

20. J'ai mentionné ailleurs (Vaillancourt, 1991c) qu'à la différence d'autres analystes, je suis davantage frappé par les éléments de rupture que par les éléments de continuité, tant sur le plan social que sur le plan constitutionnel, dans le passage du gouvernement Lesage à celui de l'Union nationale de Johnson ou de Bertrand.

21. La première recommandation du volume I du Rapport de la Commission Castonguay-Nepveu consacré à L'assurance-maladie (Castonguay et Nepveu, 1967), publié à la fin de l'été 1967, préconisait l'instauration d'un programme universel d'assurance-santé sur le modèle du programme fédéral de medicare. Mais Daniel Johnson était réticent. Il aurait préféré un modèle plus sélectif de services médicaux gratuits pour les socio-économiquement pauvres comme aux États-Unis. C'est la raison pour laquelle le dossier de I'assurance-maladie a bougé lentement entre 1967 et 1970 . Je suis reconnaissant à Mme Madeleine Blanchet, qui travaillait au sein de la Commission Castonguay à la fin des années 60 , de m'avoir sensibilisé à ce point dans une conversation téléphonique le 27 novembre 1991. Malcom Taylor y fait référence dans un chapitre consacré au Québec dans son ouvrage sur l'assurance-santé (Taylor, 1978 : chap. 7). Quant à l'ancien ministre Jean-Paul Cloutier, il a insisté dans l'entrevue qu'il m'a donnée sur la lenteur des préparatifs nécessaires pour que le Québec soit prêt à démarrer l'assurance-maladie (Cloutier, 1991).

22. Les principaux collaborateurs de René Lévesque au ministère de la Famille et du Bien-être social ont eu l'impression de pouvoir continuer sous Cloutier les réformes amorcées avec Lévesque (Marier, 1990a, 1990b ; Escojido, 1991 ; Beausoleil, 1989, 1990).

23. Comme je l'ai expliqué ailleurs (Vaillancourt, 1991b), une certaine complicité se développa entre Cloutier et Lévesque à l'époque. Cette complicité était palpable à la Commission parlementaire sur l'aide sociale à I'automne de 1969 (Cloutier, 1991 ; Escojido, 1991).

24. De l'année fiscale 1965-1966 à l'année fiscale 1968-1969, les contributions financières du ministère de la Famille et du Bien-être social à 43 agences de service social sont passées de 6,8 à 12,8 millions de dollars (MFBES, 1968).

25. Les entrevues avec Gilles Beausoleil (1989, 1990), Roger Marier (1990a, 1990b), André Escojido (1991), Jean-Paul Cloutier (1991) et Denis Fortin (1990) m'ont aidé à me faire une opinion personnelle sur le lent cheminement du dossier de l'aide sociale au cours de ces années. L'équipe de Famille et Bien-être social croyait à l'importance d'une réforme qui miserait sur la reconnaissance du droit à l'aide sociale dans le sens des recommandations du rapport Boucher et elle préparait des esquisses en conséquence. Mais lorsque ces esquisses étaient discutées au cabinet, la question qui était constamment soulevée par le ministre des Finances était celle des barèmes et des coûts. Les documents durent être refaits à plusieurs reprises. Le cabinet exigea même que la réglementation (comprenant les barèmes) soit connue avant de donner son approbation au projet de loi.

26. À la fin des années 60 , la question des procédures d'appel, que l'on tarde à mettre en place au Québec (comme en Ontario), est un point délicat qui suscite 
de l'inquiétude et de l'agacement dans l'équipe de Joe Willard à Santé et Bienêtre à Ottawa (Willard, 1969f, 1969g, 1969j ; Munro, 1969a, 1969b, 1969c).

27. Pour Des Byrne, la situation était semblable dans plusieurs provinces au début du RAPC : "When CAP started, it pretty well matched the services that provinces were providing and the target populations were within CAP authority » (Byrne, $1990: 20$ ).

28. Ce changement apparaît dans les rapports annuels du RAPC. Au chapitre de la rubrique "soins médicaux », les sommes reçues par le Québec dans le cadre du RAPC étaient de 11,9 millions de dollars en 1969-1970, de 11,5 millions en 1970-1971 et de un million en 1971-1972. En 1972-1973, le Québec ne reçoit plus rien à cette rubrique. À partir de 1974, il reçoit de nouveau des fonds pour partager les coûts de l'assistance-médicaments et il reste à l'avant-garde dans ce domaine jusqu'au début des années 80 (MSNBES, 1970, 1971, 1972, 1973, 1974, 1982).

29. Le rapport Boucher était lui-même une des sources d'inspiration du RAPC (Vaillancourt, 1992a).

30. Mais les augmentations de l'assistance publique au Québec sont amorties de 1972 à 1974, comme en témoignent également les chiffres du tableau de la page 26, en raison de l'impact de la réforme de l'assurance-chômage fédérale de 1971.

31. J'ai avancé ailleurs (Vaillancourt, 1991c) que le virage de 1973 avait été davantage celui du Québec (et de Castonguay) que celui du fédéral (et de Lalonde). De son côté, Aubert Ouellet, très proche de Castonguay à l'époque, explique le tournant de 1973 de la façon suivante : "Disons que c'était un accommodement. Ce qui nous intéressait, nous, c'était la capacité du gouvernement du Québec d'établir les politiques qui lui semblaient les plus favorables aux Québécois. Alors, dans ce sens-là, nous étions très axés sur les résultats. Les principaux moyens que nous avons considérés, ç'a été la modification de la Constitution pour que tout le secteur des politiques sociales devienne la responsabilité des provinces qui le désiraient. Ç'a été la première approche. II nous est apparu assez rapidement qu'on risquait bien d'en discuter assez longtemps sans qu'aucune modification ne soit faite. [...] II nous est apparu assez clair que le gouvernement fédéral n'abandonnerait jamais, n'abandonnerait pas dans l'immédiat à tout le moins, sa volonté de demeurer en contact avec chaque citoyen ou avec chaque famille, par le biais du versement de chèques mensuels qui rentrent dans la famille. II nous est apparu assez clairement que le gouvernement fédéral ne laisserait pas ce morceau-là. Alors, nous nous sommes dit : Étant donné qu'il semble y avoir un mur, que par ailleurs les objectifs poursuivis nous semblent bien importants, est-ce que l'on peut, tout en respectant cette volonté ferme du gouvernement fédéral, établir des mécanismes et des ententes qui nous permettraient quand même de poursuivre nos objectifs ? (Ouellet, 1989 : 8). Quant à Jacques Brunet, sous-ministre au MAS à l'époque, il résume la période de Castonguay de façon laconique : «La bataille sous Castonguay, c'est surtout une bataille pour rapatrier. Mais ça n'a pas marché beaucoup. On n'a pas fait de gros gains pendant cette période-là au point de vue du rapatriement et la grosse réforme s'est faite surtout au Québec dans les lois québécoises » (Brunet, $1990: 5)$.

32. La question du revenu minimum garanti s'est retrouvée de nouveau au cœur de certaines discussions fédérales-provinciales au cours de la « révision de la sécurité sociale », en 1975 et 1976. Mais rien de concret n'est sorti de ces discussions 
qui ont avorté dès le moment où a été abordée la question du partage des responsabilités. Quelques années plus tard, en 1979, le Québec décida d'instaurer un programme de Supplément au revenu de travail (SUPRET) pour les pauvres qui travaillent. À ce moment-là, des négociations furent entreprises avec le gouvernement fédéral pour obtenir le partage des coûts du SUPRET en vertu du RAPC. Mais ces négociations échouèrent et le Québec dut financer $100 \%$ des coûts de son nouveau programme (Duperré, 1988).

33. Plusieurs personnes interviewées ont insisté sur la différence entre le Québec et les autres provinces. Voici comment Aubert Ouellet exprime ce constat : "Un des arguments qui est souvent utilisé par les autres provinces, on nous I'a dit à de multiples reprises, pour faire accepter des choses auprès de leur gouvernement, c'est l'argument que le gouvernement fédéral va en payer la moitié. Alors, évidemment, $c^{\prime}$ est toujours plus facile de décider lorsque vous payez la moitié de la note. Je dois dire qu'au Québec on n'a jamais utilisé ces arguments-là, à ma connaissance. On a essayé vraiment d'établir les services qui nous apparaissaient prioritaires. Et, ensuite, au niveau des modalités de financement, on a essayé d'aller chercher le maximum. Mais le fait qu'Ottawa partage $50 \%$ de ces services n'a jamais été pour nous une considération importante dans la détermination des services qui devaient être mis en place. Et en ce sens-là, le Québec fonctionnait différemment des autres provinces» (Ouellet, $1988: 13$ ). Le même constat a été fait par d'autres personnes interviewées (Fonctionnaire fédéral 1, 1988 ; Duperré, 1988 ; Bédard, 1990).

34. Dans un mémo interne consacré au RAPC le 12 mai 1972, Gilles Pépin de Santé et Bien-être Canada signale que l'approche intégrée du chapitre 48 pose des problèmes d'interfaces avec le RAPC. Par exemple, il se demande comment il sera possible de distinguer, dans les activités des personnels des services intégrés, la portion qui relève des services sociaux (et du RAPC) et celle qui relève de la santé (et pas du RAPC) (Pépin, 1972).

35. André Bédard se souvient de douzaines et de douzaines de rencontres avec les fonctionnaires fédéraux au cours des années 70, uniquement pour négocier au sujet des services sociaux en milieu scolaire et hospitalier. Et «ça ne se réglait pas [...] Ils disaient que ça c'était des coûts de santé et des coûts scolaires » (Bédard, 1990 : 15).

36. Dans une riche entrevue faite avec Jean-Claude Deschênes (1990), sous-ministre au MAS à la fin des années 70 et au début des années 80, ce dernier fait une analyse sévère des implications bureaucratiques tatillonnes amenées par le RAPC dans I'administration des services sociaux, comparativement aux contraintes administratives plus légères dans le domaine de la santé régies depuis 1977 par la formule du Financement des programmes établis (Canada, 1977).

37. Dans le domaine des garderies, le Québec a profité en 1974 d'une brèche faite par le gouvernement du Manitoba en 1973. En effet, c'est le gouvernement du Manitoba qui avait obtenu du fédéral une entente administrative qui faisait une interprétation libérale du concept de " proximité du besoin » pour les services de bien-être auxquels étaient associés les services de garde. À partir de 1973 toutefois, le gouvernement fédéral allait se montrer moins flexible pour négocier de telles ententes administratives à partir du RAPC. Je suis redevable à Michael Mendelson de m'avoir communiqué ces renseignements (Mendelson, 1988).

38. Dans le cas des maisons d'hébergement pour femmes victimes de violence qui se sont développées à partir de la fin des années 70, les négociations QuébecOttawa sur l'éventuel partage des coûts à l'intérieur du RAPC achoppaient 
toujours à l'époque. André Bédard s'en souvient : "On a toujours frappé un mur! Non pas parce que le fédéral ne voulait pas. Il le voulait, mais à ses conditions qui sont les conditions du RAPC. [...] Pour le fédéral, il faut qu'elles [les femmes violentées] remplissent la petite formule. Ç'a été discuté à diverses reprises...» (Bédard, 1990 : 17).

39. Les services de garde étaient considérés comme un " service de bien-être » et la proximité du besoin était prise en considération. Le Québec utilisait un test de revenus, « mais il n'était pas comme celui du fédéral ; $c^{\prime}$ est-à-dire que le seuil en haut duquel le fédéral partageait n'était pas le même au provincial » (Bédard, $1990: 5)$.

40. C'est à partir de leur partage $d^{\prime}$ 'une vision plus progressiste des services sociaux et de la sécurité du revenu que les membres de la délégation québécoise, de façon générale, s'entendaient bien, dans les réunions de la Révision de la sécurité sociale de 1973 à 1978, avec ceux et celles des autres provinces et même du gouvernement fédéral qui avaient une perspective progressiste. Ce constat a été fait en entrevues autant par des gens du Manitoba (Mendelson, 1988 ; Ryant, 1988) et du fédéral (Osborne)

\section{Références bibliographiques (comprenant les entrevues)}

BEAUSOLEIL, G. (1989). Entrevue faite par Yves Vaillancourt avec Gilles Beausoleil à Montréal le 17 novembre 1989, transcription validée, 6 p.

BEAUSOLEIL, G. (1990). Entrevue faite par Yves Vaillancourt avec Gilles Beausoleil à Montréal le 12 juin 1990, transcription validée, 17 p.

BÉDARD, A. (1990). Entrevue faite par Yves Vaillancourt avec André Bédard à SainteFoy le 13 juin 1990, transcription validée, 38 p.

BRUNET, J. (1990). Entrevue faite par Yves Vaillancourt avec Jacques Brunet à Québec le 6 juin 1990, transcription validée, 23 p.

BYRNE, D. J. (1990). Entrevue faite par Yves Vaillancourt avec Desmond J. Byrne à Ottawa le 27 juin 1990, transcription validée, 26 p.

CANADA (1966). Régime d'assistance publique du Canada, dans Statuts du Canada, 1966-1967, chap. 45.

CANADA (1977). Loi de 1977 sur les accords fiscaux entre le gouvernement fédéral et les provinces et sur le financement des programmes établis, dans Statuts du Canada, 1977, chap. 10.

CANADA (1981). Le fédéralisme fiscal au Canada, Rapport du Groupe de travail parlementaire sur les accords fiscaux entre le gouvernement fédéral et les provinces, Ottawa, Chambre des communes, $230 \mathrm{p}$.

CARTER, F. A. G. (1971). Carter au Cabinet, 29 avril, 3 p. ANC, RG 29/85-86/343, vol. 28, dos. 3301-3-C8, par. 2.

Castonguay, C. et G. NePVeu (1967). Rapport de la Commission d'enquête sur la santé et le bien-être social, vol. I : L'assurance-maladie. Québec : Gouvernement du Québec. 
CAStOnguay, C. et G. NePVEU (1971a). Rapport de la Commission d'enquête sur la santé et le bien-être social, vol. V : La sécurité du revenu. Québec : Gouvernement du Québec, tome I.

CAstonguay, C. et G. NePVEU (1971b). Rapport de la Commission d'enquête sur la santé et le bien-être social, vol. V : La sécurité du revenu. Québec : Gouvernement du Québec, tome II.

ClOUTIER, J.-P. (1991). Entrevue faite par Yves Vaillancourt avec Jean-Paul Cloutier, à Québec, le 27 février 1991, transcription validée, 35 p.

CONSEIL NATIONAL DE BIEN-ÊTRE SOCIAL (CNBES) (1978). Le gouvernement fédéral et les services sociaux. Ottawa : CNBES, mars, $20 \mathrm{p}$.

CRAGG, N.F. (1972). Cragg à Brunet, 15 décembre, 2 p. ANC, RG 19, vol. 5514, dos. 5646-5, par. 6.

DESCHÊNES, J.-C. (1990). Entrevue faite par Yves Vaillancourt avec Jean-Claude Deschênes à Montréal le 31 mai 1990, transcription validée, 25 p.

DUPERRÉ, T. (1976). "État des relations fédérales-provinciales », dans MAS, Dossiers sécurité du revenu, Québec, 26 novembre, Planification de la sécurité du revenu, p. 51-70.

DUPERRÉ, T. (1987). La perspective fédérale-provinciale, Annexe $\mathrm{n}^{\circ} 32$ du Rapport de la Commission d'enquête sur les services de santé et les services sociaux, Québec : Les Publications du Québec, 42 p.

DUPERRÉ, T. (1988). Entrevue faite par Yves Vaillancourt avec Thomas Duperré à Québec le 18 mai 1988, transcription validée, 13 p.

DUPERRÉ, T. (1990). Entrevue faite par Yves Vaillancourt avec Thomas Duperré à Québec le 18 avril 1990, transcription validée, 5 p.

ESCOJIDO, A. (1991). Entrevue faite par Yves Vaillancourt avec André Escojido à Québec le 27 février 1991, transcription validée, 34 p.

FONCTIONNAIRE FÉDÉRAL [FF1]. Entrevue faite par Yves Vaillancourt avec un fonctionnaire fédéral au printemps 1990, transcription non validée, 34 p.

FORTIN, D. (1990). Entrevue faite par Yves Vaillancourt avec Denis Fortin à Québec le 6 juin 1990, transcription validée, 26 p.

GARNER, J. (1973). Garner à Goyette, 28 mars, 9 p. ANC, RG 19, vol. 5514, dos. 5646-5-2, par. 6.

HEPWORTH, P. (1988). Entrevue faite par Yves Vaillancourt avec Philip Hepworth le 25 mars 1988 à Ottawa, transcription validée, 16 p.

KNIGHT, N. (1990). Entrevue faite par Yves Vaillancourt avec Norman Knight à Ottawa le 20 juin 1990, transcription validée, 14 p.

LALONDE, M. (1973). Document de travail sur la sécurité sociale au Canada. Ottawa : Gouvernement du Canada, 18 avril, 57 p.

LEACH, D.J. (1971). Record of Cabinet Decision re Proposals for Amending the CAP, Meeting of May 6, 7 mai, 2 p. ANC, RG 29/85-86/343, vol. 28, dos. 3301-3C8, par. 2.

MARIER, R. (1969). «Les objectifs sociaux du Québec », Canadian Public Administration / Administration publique du Canada, vol. 12, $\mathrm{n}^{\circ} 2$ : 181-197.

MARIER, R. (1990a). Entrevue faite par Yves Vaillancourt avec Roger Marier à Québec le 17 mai 1990, transcription validée, 16 p.

MARIER, R. (1990b). Entrevue faite par Yves Vaillancourt avec Roger Marier à Québec le 20 juillet 1990, transcription validée, 20 p. 
MENDELSON, M. (1988). Entrevue faite par Yves Vaillancourt avec Michael Mendelson à Winnipeg le 17 mars 1988, transcription validée, $13 \mathrm{p}$.

MINISTÈRE DE LA FAMILLE ET DU BIEN-ÊTRE SOCIAL (MFBES) (1968). Contributions financières du ministère aux agences sociales. Québec : Gouvernement du Québec. Archives de Roger Marier, $3 \mathrm{p}$

MINISTÈRE DE LA FAMILLE ET DU BIEN-ÊTRE SOCIAL (MFBES) (1970). Rapport annuel 1969-1970. Québec : Gouvernement du Québec, 246 p.

MINISTÈre DE LA SANTÉ NATIONALE ET DU BIEN-ÊTRE SOCIAL (MSNBES) (1967 à 1982). Régime d'assistance publique du Canada. Rapport annuel 1966-1967, Ottawa, MSNBES, 38 p. ainsi que les années ultérieures jusqu'en 1981-1982.

MunRO, J. (1968). Relation of the CAP to Institutional Care and Services for Juvenile Delinquents, non daté, 4 p. ANC, RG 29, vol. 1526, dos. 201-16-1A.

Munro, J. (1969a). Munro à Cloutier, 17 avril, 2 p. ANC, RG 29, vol. 1524, dos. 201-16-1.

MunRO, J. (1969b). Munro à Cloutier, 28 mai, 1 p. ANC, RG 29, vol. 2115, dos. 234-8.

MunRO, J. (1969c). Munro à Cloutier, 24 septembre, 2 p. ANC, RG 29, vol. 2115, dos. 23-4-8.

MunRO, J. (1970). La sécurité de revenu au Canada. Ottawa : ministère de la Santé nationale et du Bien-être social, $109 \mathrm{p}$.

Munro, J. (1971a). Munro au Cabinet, 2 février, 5 p. + annexe de 6 p. ANC, RG 19/85-86/343, vol. 28, dos. 3301-3-C6.

MunRO, J. (1971b). Munro au Cabinet, 26 avril, 5 p. ANC, RG 29/85-86/343, vol. 28, dos. 3301-3-C8, par. 2.

MunRO, J. (1971c). Munro au Cabinet, 3 mai, 5 p. ANC, RG 29/85-86/343, vol. 28, dos. 3301-3-C8, par. 2.

Munro, J. (1971d). Munro au Cabinet, 11 mai, 3 p. ANC, RG 29/85-86/343, vol. 28, dos. 3301-3-C8, par. 2.

Munro, J. (1971e). Munro au Cabinet, 31 mai, 3 p. ANC, RG 29/85-86/343, vol. 28, dos. 3301-3-C8, par. 2.

OSBORNE, J.E. (1986). "Evolution of the Canada Assistance Plan (CAP)», dans Canada, Task Force on Program Review, Service to the Public. Canada Assistance Plan, Ottawa : Minister of Supply and Services, annexe 3, 57-92.

OSBORNE, J.E. (1990). Entrevue faite par Yves Vaillancourt avec John E. Osborne à Nepean le 21 juin 1990, transcription validée, 22 p.

OuELLET, A. (1988). Entrevue faite par Yves Vaillancourt avec Aubert Ouellet, le 23 juin 1988 à Québec, transcription validée, 18 p.

PÉPIN, G. (1972). Canada Assistance Plan, 12 mai, 3 p. ANC, RG 29, vol. 1605, dos. 7.

PréFOntaine, N. (1971). Proposals for Amending the CAP, Comité du cabinet sur la politique sociale, réunion du 4 mai, 2 p. + annexe de 3 p. ANC, RG 29/85-86/ 343, vol. 28, dos. 3301-3-C8, par. 2.

QUÉBEC (1964). Loi de l'assistance publique, dans Lois refondues du Québec, chap. 216.

QUÉBEC (1969). Loi de l'aide sociale, dans Lois du Québec, 1969, chap. 63.

QUÉBEC (1970). Loi sur l'assurance-maladie, dans Lois du Québec, 1970, chap. 37. 
QUÉBEC (1971). Loi sur les services de santé et les services sociaux, dans Lois du Québec, 1971, chap. 48.

QUÉBEC (1977). Loi sur la protection de la jeunesse, dans Lois du Québec, 1977, chap. 20.

QUÉBEC (1978). Loi assurant l'exercice des droits des personnes handicapées, dans Lois du Québec, 1978.

QUÉBEC (1979). Loi sur les services de garde à l'enfance, dans Lois du Québec, chap. 85.

QUÉBEC et CANADA (1967). Accord intervenu dans le cadre du Régime d'assistance publique du Canada, Québec, texte dactylographié, 8 p.

RoulEAU, R. (1990). Entrevue faite par Yves Vaillancourt avec Réal Rouleau à Orléans le 24 juillet 1990, transcription validée, 17 p.

RYANT, J. (1988). Entrevue faite par Yves Vaillancourt avec Joseph Ryant à Winnipeg le 11 mars 1988, transcription non validée, $15 \mathrm{p}$.

TAYLOR, M.G. (1978). Health Insurance and Canadian Public Policy. Montréal et Kingston : McGill Queen's, 473 p.

VAILLANCOURT, Y. (1991a). "Le Régime d'assistance publique du Canada : revue de la littérature québécoise et canadienne», Canadian Review of Social Policy / Revue canadienne de politique sociale, $\mathrm{n}^{\circ}$ 27, mai : 20-33.

VAILLANCOURT, Y. (1991b). «René Lévesque et les politiques sociales dans les années $60 »$, Nouvelles pratiques sociales, vol. 4, n 1, printemps : 153-166.

VAILLANCOURT, Y. (1991c). «Un bilan québécois des quinze premières années du Régime d'assistance publique du Canada (1966-1981) : la dimension constitutionnelle », Nouvelles pratiques sociales, vol. 4, n 2, automne : 115-146.

VAILLANCOURT, Y. (1992a). " Les origines du RAPC examinées en mettant l'accent sur le rôle du ministère de la Santé nationale et du Bien-être social : une lecture québécoise (1960-1966) », article soumis à la Canadian Review of Social Policy / Revue canadienne de politique sociale, $\mathrm{n}^{\circ} 29$, mai.

VAILLANCOURT, Y. (1992b). « Les origines du RAPC examinées en mettant l'accent sur le rôle du ministère des Finances : une lecture québécoise (1960-1966) ", article soumis à la Canadian Review of Social Policy / Revue canadienne de politique sociale, $\mathrm{n}^{\circ}$ 30, novembre.

WILLARD, J.W. (1969a). Willard à Robertson, 21 janvier, 2 p. ANC, RG 29, vol. 1524, dos. 201-16-1.

WILLARD, J.W. (1969b). Willard à Munro, 13 février, 1 p. + annexe. ANC, RG 29, vol. 2115, dos. 23-4-7.

WILLARD, J.W. (1969c). Willard à Marier, 17 février, copie anglaise, 2 p. ANC, RG 29, vol. 1524, dos. 201-16-1.

WILLARD, J.W. (1969d). Willard à Munro : Institutional Care and Services for Juvenile Delinquents, 19 février, 3 p. ANC, RG 29, vol. 1524, dos. 201-16-1.

WILLARD, J.W. (1969e). Willard à Munro, 25 février, 4 p. ANC, RG 29, vol. 2115, dos. 23-4-7.

WILLARD, J.W. (1969f). Willard à Munro, 16 avril, 2 p. + annexe de 4 p. ANC, RG 29, vol. 1526, dos. 201-16-1A.

WILLARD, J.W. (1969g). Willard à Munro, 23 avril, 5 p. ANC, RG 29, vol. 1526, dos. 201-16-1A.

WILLARD, J.W. (1969h). Willard à Clarkson, 2 mai, 3 p. ANC, RG 29, vol. 1524, dos. 201-16-1. 
WILLARD, J.W. (1969i). Willard à Marier, 2 septembre, 2 p. ANC, RG 29, vol. 2115, dos. 23-4-8.

WILLARD, J.W. (1969j). Willard Report, Ottawa, Bureau du Conseil privé, volume I (rapport jamais rendu public).

WILLARD, J.W. (1969k). Willard Report, Ottawa, Bureau du Conseil privé, volume II (rapport jamais rendu public).

WILLARD, J.W. (1970). "Mémoire du ministère de la Santé et du Bien-être social du Canada » devant le Comité sénatorial (Croll) sur la pauvreté dans Procèsverbaux du Sénat, mardi 24 février et jeudi 26 février, $23: 5$ à $23: 97$.

YZERMAN, R. (1988). Entrevue faite par Yves Vaillancourt avec Ron Yzerman à Ottawa le 8 juin 1988, transcription validée, 20 p. 